\title{
Correction to: Epidemiological and clinical characteristics of patients with suspected COVID-19 admitted in Metro Manila, Philippines
}

Eumelia P. Salva', Jose Benito Villarama', Edmundo B. Lopez', Ana Ria Sayo ${ }^{1}$, Annavi Marie G. Villanueva², Tansy Edwards ${ }^{2,3}$, Su Myat Han ${ }^{2,4}$, Shuichi Suzuki ${ }^{2}$, Xerxes Seposo ${ }^{2}$, Koya Ariyoshi ${ }^{2,4}$ and Chris Smith ${ }^{2,5^{*}}$

\section{Correction to: Trop Med Health 48, 51 (2020) https://doi.org/10.1186/s41182-020-00241-8}

Following the publication of the original article [1], it was noted that the light red color is missing from the histogram in Fig. 1. The corrected figure has been shown below:

\section{Author details}

'San Lazaro Hospital, Manila, Philippines. ${ }^{2}$ School of Tropical Medicine and Global Health, Nagasaki University, Nagasaki, Japan. ${ }^{3}$ MRC Tropical Epidemiology Group, London School of Hygiene and Tropical Medicine, London, UK. ${ }^{4}$ Institute of Tropical Medicine, Nagasaki University, Nagasaki 852-8523, Japan. ${ }^{5}$ Faculty of Infectious and Tropical Diseases, London School of Hygiene and Tropical Medicine, London, UK.

Published online: 07 July 2020

\section{Reference}

1. Salva EP, et al. Epidemiological and clinical characteristics of patients with suspected COVID-19 admitted in Metro Manila, Philippines. Trop Med Health. 2020:48:51.

\footnotetext{
The original article can be found online at https://doi.org/10.1186/s41182020-00241-8

* Correspondence: christopher.smith@lshtm.ac.uk

${ }^{2}$ School of Tropical Medicine and Global Health, Nagasaki University,

Nagasaki, Japan

${ }^{5}$ Faculty of Infectious and Tropical Diseases, London School of Hygiene and

Tropical Medicine, London, UK

Full list of author information is available at the end of the article
}

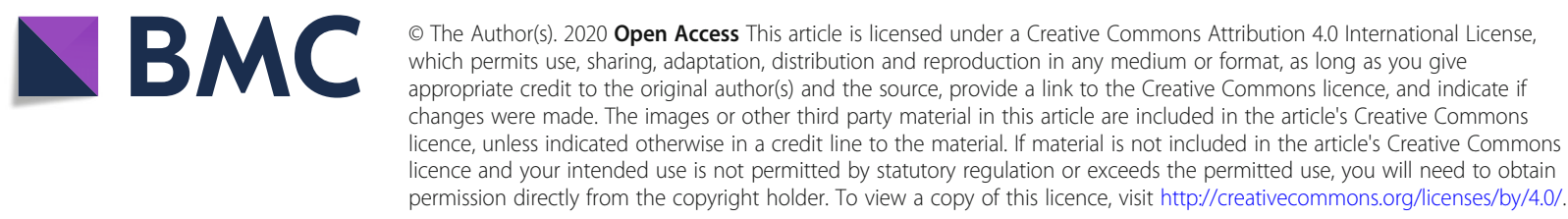




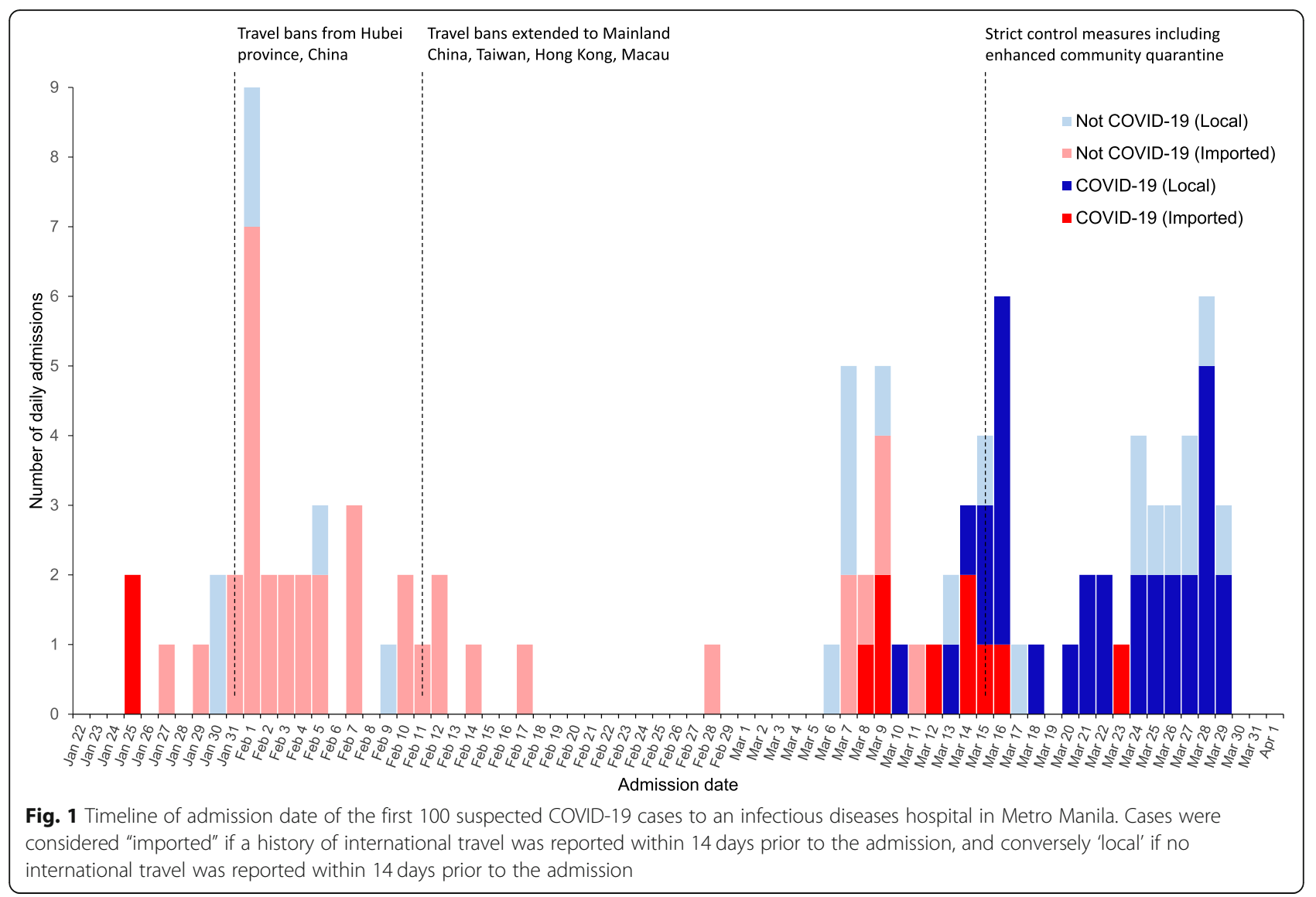

